\title{
Nas "Filas de Espera": Tempo entre Submissão e Aceitação de Manuscritos em Periódicos Brasileiros de Psicologia
}

\author{
Jean Von Hohendorff ${ }^{1}$ \\ Diogo Araújo DeSousa \\ Departamento de Psicologia da Universidade Tiradentes, Aracaju, SE, Brasil \\ Anderson Siqueira Pereira \\ Silvia Helena Koller \\ Programa de Pós-Graduação em Psicologia da Universidade Federal do Rio Grande do Sul, \\ Porto Alegre, RS, Brasil
}

\section{Resumo}

Revistas científicas são um dos principais veículos para a disseminação de achados científicos tendo um papel crucial no desenvolvimento da ciência. No entanto, tais objetivos exigem um processo editorial eficiente. O objetivo desse estudo foi descrever a média de tempo entre submissão e aceitação de manuscritos em revistas brasileiras de alta qualidade em Psicologia e investigar associações entre o tempo de submissão/aceitação e aspectos do processo editorial. Foram selecionados 44 periódicos dos estratos mais altos do Qualis CAPES da área de Psicologia. Foi consultado o tempo entre as datas de submissão e aceitação dos artigos disponíveis online nos quatro últimos números de cada revista $(n=2.580)$. A média de tempo foi de 235,37 dias $(D P=192,95)$. Quanto mais alto o estrato Qualis, maior o tempo entre submissão/aceitação. Quanto maior o número de membros no corpo editorial, menor o tempo entre submissão/aceitação. O tempo entre submissão/aceitação foi menor nas revistas de associações/conselhos em comparação às revistas de universidades/faculdades. Os resultados são discutidos enfatizando o impacto de processos editoriais na divulgação do conhecimento e na avaliação de pesquisadores e revistas científicas. Sugestões são oferecidas com a finalidade de tornar mais eficaz o processo editorial.

Palavras-chave: Políticas editoriais, indicadores de produção científica, artigo de revista.

\section{On "Waiting Lines": Time between Submission and Acceptance Dates of Manuscripts in Brazilian Psychology Journals}

\begin{abstract}
Scientific journal are a major mean for disseminating scientific findings, and play a crucial role in the development and updating of science. However these goals require an efficient editorial process. The aims of this study were to describe the average time between submission and acceptance of manuscripts in high quality Brazilian journals in Psychology and to investigate associations between the submission/ acceptance time and aspects of the editorial process. We selected 44 journals from the highest strata of the Psychology Qualis CAPES. Published articles available online in the last four numbers of each journal were consulted in regard to the declared time between the dates of submission and acceptance $(N=$ $2,580)$. The average submission/acceptance time was 235.37 days $(S D=192.95)$. The higher the Qualis
\end{abstract}

Endereço para correspondência: Universidade Federal do Rio Grande do Sul, Instituto de Psicologia, Rua Ramiro Barcelos, 2600/104, Porto Alegre, RS, Brasil 90035-003. E-mail: jhohendorff@gmail.com 
stratum of the journal, the longer the submission/acceptance time. The higher the number of members in the editorial board, the faster submission/acceptance time. The submission/acceptance time was faster in journals of associations/councils as compared to journals of public and private universities/colleges. Results are discussed emphasizing the impact of a long editorial process in the dissemination of science and the assessment of researchers and scientific journals. Suggestions are offered to make the editorial process more effective.

Keywords: Editorial polices, scientific publication indicators, journal article.

\section{En la "Fila de Espera": Tiempo entre la Sumisión y la Aceptación de Manuscritos en Periódicos Brasileros de Psicología}

\section{Resumen}

Revistas científicas son uno de los principales vehículos para la diseminación de los hallazgos científicos y tienen un papel crucial en el desarrollo de la ciencia. Sin embargo, tales objetivos exigen un proceso editorial eficiente. El objetivo del estudio fue describir la media del tiempo entre la sumisión y aceptación de manuscritos en revistas brasileras de alta cualidad en Psicología, e investigar asociaciones entre el tiempo de sumisión/aceptación y aspectos del proceso editorial. Fueron seleccionados 44 periódicos de los estratos más altos del Qualis Capes de Psicología. Fueron consultados el tiempo entre las fechas de sumisión y aceptación de los artículos disponibles online en los cuatro últimos números de cada revista $(n=2.580)$. La media de tiempo fue de 235,37 días $(S D=192,95)$. Cuanto más alto el estrato Qualis, mayor el tiempo de sumisión/aceptación. Cuanto más miembros en el grupo editorial, menor el tiempo de sumisión/aceptación. El tiempo de sumisión/aceptación fue menor en las revisas de asociaciones/ consejos en comparación a las de universidades/facultades. Los resultados son discutidos enfatizando el impacto de procesos editoriales en la divulgación del conocimiento y evaluación de investigadores y revistas científicas. Sugerencias son ofrecidas con la finalidad de volver más eficaz el proceso editorial.

Palabras clave: Políticas editoriales, indicadores de producción científica, artículo de revista.

O objetivo da ciência pode ser identificado primordialmente como o de gerar continuamente conhecimento atualizado e inovador, cujo acúmulo promova melhorias na qualidade de vida das pessoas (Trzesniak \& Koller, 2009). As revistas científicas são um dos principais meios para apresentar este corpo acumulado de conhecimento à comunidade científica. As revistas são também denominadas periódicos científicos, enfatizando a perspectiva de divulgar resultados mais atualizados das pesquisas e da produção de conhecimento disponível (Sabadini, Sampaio, \& Nascimento, 2009). Uma das categorias de publicação mais comuns em periódicos científicos são os artigos originais. Artigos originais devem ser frutos de estudos não publicados previamente, no geral revisados por pares, e devem contribuir para o avanço do conhecimento na ciência. A revisão por pares é adotada em amplo número de periódicos com o objetivo de que o manuscri- to seja cuidadosamente avaliado por especialistas em sua área antes da divulgação. Durante o processo de revisão, o manuscrito é considerado um documento confidencial e privilegiado, sendo geralmente acessado somente pelos editores e revisores (American Psychological Association [APA], 2010). Embora todas estas questões sejam relevantes, o cenário das publicações brasileiras, com relação à atualidade das publicações tem sido questionado, especialmente devido à demora no processo submissão-publicação dos manuscritos.

O processo de revisão de um manuscrito inicia-se após a sua submissão pelos autores. A partir da submissão, editores e editores associados geralmente designam revisores que emitirão pareceres sobre o manuscrito, recomendando ou não sua publicação ou sugerindo reformulações. Muitos periódicos utilizam um processo de revisão "duplo-cego" (double blind review), no 
qual a identidade dos autores não é revelada aos revisores e vice-versa, com o objetivo de evitar vieses pessoais na avaliação. O tempo para que um processo de revisão seja concluído é estimado pela APA (2010) em aproximadamente dois a três meses, porém, esse tempo dependerá do tamanho e complexidade do manuscrito e do número de revisores envolvidos. A decisão final pela publicação ou não do manuscrito cabe aos editores e/ou editores associados da revista (APA, 2010).

No Brasil, a Coordenação de Aperfeiçoamento de Pessoal de Nível Superior (CAPES) avalia as revistas científicas nas quais pesquisadores brasileiros publicam artigos originais (Yamamoto \& Costa, 2009). Para tanto, existem comissões dentro de cada área de conhecimento que classificam as revistas em oito estratos: $C$, B5, B4, B3, B2, B1, A2, e A1 (em ordem crescente de maior qualidade e abrangência). Cada comissão tem a liberdade de definir critérios para a avaliação das revistas. A comissão de avaliação em Psicologia da CAPES utiliza critérios integrados para avaliar as revistas brasileiras e estrangeiras da área de Psicologia nestes estratos. Os requisitos mínimos para a inclusão das revistas nos estratos incluem: possuir ISSN (International Standard Serial Number), conselho editorial, normas de submissão, periodicidade mínima semestral, avaliação por pares, existência de resumos e abstract nos artigos, e data de recebimento e aceite dos artigos (CAPES, 2013). Com o preenchimento destes requisitos mínimos, as revistas são avaliadas a partir dos indexadores em que estão presentes. Uma hierarquia de indexadores agrupa as revistas em cada um dos estratos (CAPES, 2013).

A avaliação de revistas de outras áreas, mas em que pesquisadores da área de Psicologia publicam (e.g., autores da Psicologia publicando em revistas da Educação), é realizada de forma diferente. Inicialmente, os critérios da Psicologia são aplicados. Caso a avaliação da revista a partir dos critérios da Psicologia seja diferente da realizada por sua área original (e.g., Educação), a avaliação de sua área original é mantida se a diferença entre as duas (e.g., Psicologia e Educação) for de menos de dois estratos. Caso essa diferença seja de mais de dois estratos, a avaliação da Psicologia é mantida com o acréscimo ou decréscimo de um estrato (CAPES, 2013).

No Brasil, o principal indexador avaliativo de periódicos científicos é a Biblioteca Científica Eletrônica Online SciELO (Scientific Electronic Library Online, ver em http://www.scielo. org). Criada em 1998, a SciELO é responsável pela "indexação, agregação, publicação e interoperabilidade de coleções em acesso aberto de periódicos científicos avaliados pelos pares, publicadas por instituições nacionais dos países ibero-americanos e da África do Sul" (Packer \& Meneghini, 2014, p. 15). Este indexador está baseado na "ideia de que o progresso da ciência implica progresso da comunicação científica, que inclui a capacidade de produzir periódicos de qualidade" (p. 19). A SciELO possui critérios de avaliação da qualidade dos periódicos para sua admissão e permanência no indexador. Há critérios comuns para periódicos de diferentes áreas, bem como critérios específicos para cada área. Dentre os critérios comuns adotados pela SciELO, diversos estão relacionados ao processo editorial: pontualidade, periodicidade e quantidade de artigos publicados por ano, tempo de processamento dos artigos, gestão editorial do processo de submissão dos artigos, e necessidade de indicação das principais datas (i.e., recebimento e aprovação) do processo de avaliação do manuscrito no artigo publicado (Montanari \& Packer, 2014). Nos últimos anos, a SciELO tem considerado como seus principais desafios a internacionalização (i.e., expansão do público dos periódicos) e profissionalização (i.e., adoção de serviços de editoração e publicação de última geração e desempenho de editores e equipes editoriais) da gestão editorial dos periódicos (Packer \& Meneghini, 2014). Além da SciELO, o Brasil conta com um portal de periódicos específico para a ciência psicológica - o portal de Periódicos Eletrônicos em Psicologia (PePSIC, ver em http://pepsic.bvsalud.org). Trata-se de uma ferramenta da Biblioteca Virtual em Saúde - Psicologia (BVS-Psi, www.bvs-psi.org.br) que também disponibiliza em acesso aberto os textos completos de revistas científicas brasileiras da psicologia e áreas afins. A inclusão de uma revista na 
BVS-Psi está condicionada ao atendimento de, no mínimo, $70 \%$ dos 33 critérios adotados pelo Latindex, bem como ao parecer de pareceristas externos quando necessário. Dentre estes critérios, alguns dizem respeito ao processo editorial: presença de avaliadores externos à entidade ou instituição editora do periódico, fornecimento de informações sobre os procedimentos utilizados para a seleção dos artigos, indicação das datas de recepção e aceitação dos artigos (para lista completa, acessar http://www.latindex.unam. $\mathrm{mx} /$ documentos/revistas_imp_por.html). Periódicos indexados na SciELO, Thomson Reuters e PsycInfo estão automaticamente aprovados para inclusão no portal PePSIC.

SciELO e PEPSIC têm recebido reconhecimento no país como importantes disseminadores do conhecimento em Psicologia. No entanto, ambos indexadores, assim como os periódicos neles indexados, enfrentam desafios operacionais (e.g., profissionalização da gestão dos periódicos e dos processos de disseminação e marketing da ciência; hierarquização e internacionalização dos periódicos brasileiros). Tais desafios têm reflexo no trabalho de pesquisadores autores, uma vez que dependem dos periódicos para a publicação dos resultados de suas pesquisas. A publicação dos resultados de uma pesquisa em um periódico científico é um compromisso social dos pesquisadores, tendo em vista que parte significativa das pesquisas em programas de pós-graduação no Brasil é financiada por fundos públicos. Além disto, programas de pós-graduação e seus pesquisadores costumam ser avaliados a partir de sua produção científica. Em relação a publicações, atualmente no Brasil existe uma sobrevalorização da quantidade de publicações em detrimento de sua relevância ou benefício acadêmico e social (Yamamoto \& Costa, 2009; Yamamoto, Tourinho, Bastos, \& Menandro, 2012). O resultado comumente é a busca pelo maior número de publicações possível, ainda que isso implique decréscimo na qualidade do material publicado.

As revistas científicas e os pesquisadores comumente são avaliados pelo impacto de suas publicações, por meio de índices como o fator de impacto e o fator $h$, respectivamente. $\mathrm{O}$ fator $h$ é calculado por meio do número de artigos publicados que tenham citações maiores ou iguais a esse número (Thomaz, Assad, \& Moreira, 2011). Por exemplo, para possuir um fator $h$ de 12, a revista e ou o(a) pesquisador(a) devem possuir pelo menos 12 artigos que tenham sido citados 12 ou mais vezes. Para calcular o fator de impacto de uma revista, pelo ISI - Web of Knowledge (http://ip-science.thomsonreuters. com) ou SCImago/Scopus (www.scimago.es), é levada em consideração a quantidade de citações recebidas naquele ano pelos artigos publicados nos dois anos anteriores, dividida pelo número de artigos publicados no mesmo período. Desta forma, a publicação em revistas mais conhecidas e com um fator de impacto maior pode render mais referências ao trabalho dos pesquisadores aumentando seu fator $h$ e sua visibilidade dentro da comunidade científica.

Índices como o fator de impacto e o fator $h$ são indicadores bibliométricos utilizados pela cientometria para investigar os fenômenos relacionados ao progresso da ciência e tecnologia. Em uma definição simplificada, a cientometria é uma área de estudo que utiliza mensuração quantitativa para "estudar a ciência da ciência" (Silva \& Bianchi, 2001, p. 6). Estes indicadores são utilizados, ainda, para a concessão de fomento às instituições e aos pesquisadores. Os indicadores bibliométricos utilizados pela cientometria tendem a ser baseados em quantidade (e.g., número de publicações, citações). O número de citações que um artigo recebe é um indicador bibliométrico quantitativo que visa a acessar sua qualidade, pois se acredita que artigos de maior qualidade recebem maior número de citações. No entanto, artigos podem receber grande número de citações críticas ou negativas. Devido a isto, considera-se que o uso estrito de indicadores bibliométricos quantitativos na cientometria não é adequado (Silva \& Bianchi, 2001). Recentemente, foi publicado o manifesto de Leiden (acessar http://www.leidenmanifesto. org para descrição detalhada do manifesto), que vem recebendo grande visibilidade. $\mathrm{O}$ manifesto, proposto por cinco experts em cientometria, possui dez princípios para a avaliação científica. A valorização de indicadores qualitiativos (e.g., avaliação qualitativa por experts) está presente em alguns destes princípios, evidenciando a necessidade de readequação dos critérios aplicados 
na cientometria quanto ao uso estrito de indicadores bibliométricos quantitativos.

Diante da pressão pela produtividade, cabe aos pesquisadores o compromisso social de publicar os resultados de sua pesquisa com qualidade, enquanto aos envolvidos no processo de revisão cabe torná-lo o mais idôneo e ágil possível visando à qualidade e atualização das publicações. A tarefa de agilizar o processo de revisão tem sido um desafio diante do crescente número de submissões e publicações. Por exemplo, dados obtidos por meio dos currículos Lattes dos 297 pesquisadores bolsistas de produtividade científica em Psicologia do Conselho Nacional de Desenvolvimento Científico e Tecnológico (CNPq) em 2011 demonstraram uma média de 15,91 artigos $(D P=10,68)$ publicados por bolsista no período de quatro anos entre 2007 e abril de 2011 (Wendt, Lisboa, DeSousa, \& Koller, 2013). Isso equivale a aproximadamente quatro artigos publicados por ano por bolsista, uma média já consideravelmente mais alta que a estimativa de 2010 de 2,26 artigos por docente em programas de pós-graduação em Psicologia para o triênio de 2007 a 2009 (esta de menos de um artigo por ano; Hutz, Rocha, Spink, \& Menandro, 2010). Além disso, embora haja variação entre os pesquisadores, se o total de bolsistas avaliados no estudo de Wendt et al. (2013) for considerado $(N=297)$, o número de publicações entre 2007 e 2011 ficou em torno de 4.725 artigos, ou seja, aproximadamente 1.181 artigos publicados por ano. Tal número leva em conta somente artigos publicados pelos pesquisadores com bolsa de produtividade em pesquisa do $\mathrm{CNPq}$ em Psicologia. Sendo assim, o número de submissões deve ser considerado um aspecto importante que impacta o tempo do processo de revisão de um artigo científico.

Diante do exposto, os objetivos deste estudo foram:

1. Estimar o tempo médio entre a submissão e aceitação de manuscritos nas revistas científicas mais bem avaliadas em Psicologia no Brasil (i.e., revistas dos mais altos estratos Qualis: A1, A2 e B1); e

2. Investigar possíveis associações entre variáveis editoriais relacionadas às revistas científicas e o tempo despendido no processo de revisão dos seus manuscritos. As variáveis investigadas foram: idade da revista, número de membros nas comissões/comitês editoriais, instituição vinculada, região geográfica e idiomas de publicação.

\section{Método}

Foi realizado um estudo quantitativo transversal a partir dos dados de artigos publicados em revistas brasileiras dos estratos Qualis A1, A2 e B1 em Psicologia.

\section{Amostra}

A amostra de publicações consultadas foi obtida nas revistas dos estratos Qualis A1 e A2 em Psicologia publicadas no Brasil ( $n=22$, sendo três revistas A1 e 19 revistas A2) no triênio 2010-2012, segundo classificação CAPES (n.d.), bem como foi sorteado aleatoriamente o mesmo número de revistas Qualis B1 em Psicologia $(n=$ $22 ; 4,59 \%$ do total de revistas B1). O sorteio foi realizado a partir de uma lista de números aleatórios gerados por software comparados aos números atribuídos às revistas Qualis B1 organizadas em ordem alfabética. Assim, foram analisados, ao final, dados de 44 revistas. A média de idade das revistas foi de 16,34 anos $(D P=11,97)$, com amplitude de três a 66 anos. A média da quantidade de membros editoriais foi de 53,32 ( $D P=$ 30,37), com amplitude de nove a 139 membros. Quanto à instituição da revista, 24 (54,5\%) são revistas vinculadas a universidades/faculdades públicas, sete (15,9\%) são vinculadas a universidades/faculdades privadas, e as $13(29,5 \%)$ restantes são vinculadas a associações/conselhos. Quanto à região geográfica, duas são do Nordeste $(4,5 \%)$, duas do Centro-Oeste $(4,5 \%)$, oito do Sul (18,2\%), e 32 do Sudeste (72,7\%). Em relação aos idiomas de publicação aceitos pelas revistas, quatro delas $(9,1 \%)$ aceitam artigos apenas em língua portuguesa, quatro $(9,1 \%)$ aceitam artigos em português e espanhol, e 29 (66,1\%) aceitam artigos em três ou mais idiomas. Para as sete revistas restantes $(15,9 \%)$ não havia especificação sobre quais idiomas são aceitos nas regras de submissão divulgadas online.

Todos os artigos publicados nessas revistas e disponíveis online em seus quatro últimos nú- 
meros $(n=2.580)$ foram consultados a fim de se obter o tempo entre a data de submissão e aceitação de cada artigo. Dos 2.580 artigos, 190 (7,4\%) foram artigos publicados nas três revistas Qualis A1; 1.052 (40,8\%) foram artigos publicados nas 19 revistas Qualis A2; e 1.338 (51,9\%) foram artigos publicados nas 22 revistas Qualis B1 selecionadas aleatoriamente.

\section{Procedimentos}

Inicialmente foi consultada a lista de revistas nos estratos Qualis CAPES A1 e A2 em Psicologia com o objetivo de verificar seu número total $(N=22)$. Em seguida, o mesmo número de revistas Qualis B1 foi aleatoriamente sorteado com o objetivo de se obter uma amostra representativa desse estrato, vista a impossibilidade de incluir todas as revistas B1 nesta amostra. As variáveis relacionadas às revistas (i.e., idade, quantidade de membros editorais, instituição vinculada, região geográfica e idiomas de publicação) foram avaliadas em conjunto com as datas de submissão, revisões e aceitação de todos os artigos ( $n$ $=2.580$ ) publicados nos últimos quatro números das 44 revistas. Todos esses dados foram extraídos a partir dos sites das revistas no período de novembro de 2014 a janeiro de 2015. Apenas dados de data (i.e., submissão, revisões e aceites) de artigos originais (empíricos ou de revisão) foram computados, excluindo-se editoriais, notas técnicas, resenhas e demais publicações.

\section{Resultados}

Considerando o total da amostra, a média de dias entre a data de submissão e a data de aceitação dos manuscritos nas revistas brasileiras nos mais altos estratos Qualis foi de 235,37 dias (DP $=192,95)$, isto é, por volta de oito meses. Em sete casos dos 2.580 avaliados, a data do aceite foi declarada como a mesma da submissão (zero dia). O máximo de tempo decorrido entre a submissão e o aceite foi de 1.492 dias, ou seja, mais de quatro anos. Mesmo após a exclusão dos casos extremos (i.e., zero dia entre submissão e aceite do manuscrito ou mais de 1.200 dias; $n=$ 14), a média de tempo é bastante similar ( $M=$ $233,07$ dias; $D P=184,55)$. A mediana de tempo foi de 183 dias (percentil 25\% = 103; percentil $75 \%=306$ ) e a moda 153 dias. A Figura 1 apresenta um histograma com a distribuição da variável na amostra.

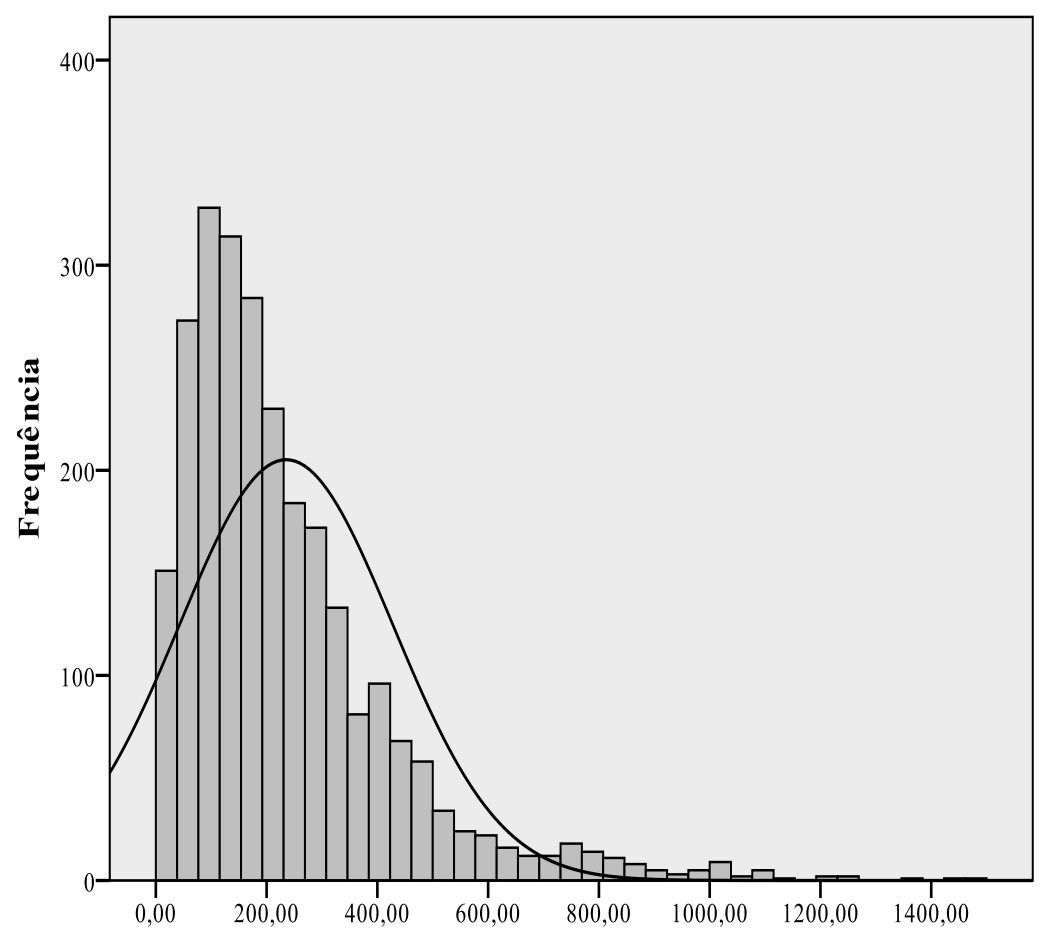

Dias entre data de submissão e aceite do manuscrito

Figura 1. Histograma com a distribuição do tempo (em dias) decorrido entre as datas de submissão e de aceite dos manuscritos avaliados na amostra. 
No entanto, é preciso considerar que o tempo entre a submissão e aceitação de um manuscrito pode ser influenciado pelo tempo que os autores levam para responder aos pareceres das revistas quando estas solicitam alterações no manuscrito como necessárias para se considerar a publicação. Assim, foi realizado um refinamento da análise considerando o tempo entre a data de submissão e a data da primeira revisão do manuscrito, ao invés da data de aceitação final, quando havia data(s) de revisão declarada(s). Essa nova análise demonstrou um tempo médio entre a data de submissão e a data da primeira revisão do manuscrito (ou aceitação, caso não fossem informadas datas de revisão) de 211,22 dias $(D P=178,90)$, cerca de sete meses.

Foi encontrada uma diferença significativa no tempo para aceitação do manuscrito em função do estrato Qualis da revista (Tabela 1). O tempo entre a data de submissão e a data de aceitação foi diretamente proporcional ao estrato Qualis da revista. Assim, artigos publicados em revistas Qualis A1 levaram mais tempo entre a submissão e aceitação (média aproximada de 12 meses) que em revistas Qualis A2 (média aproximada de 8 meses e meio), que por sua vez levaram mais tempo que em revistas Qualis B1 (média aproximada de 6 meses e meio; Tabela 1). Testes post-hoc Bonferroni demonstraram que as diferenças foram significativas entre os três estratos (para todas as comparações, $p<$ $0,001)$. Os tamanhos de efeito dessas diferenças ( $d$ de Cohen) foram de médio (entre as revistas de Qualis A1 e A2 e entre as revistas de Qualis A2 e B1) a grande (entre as revistas de Qualis A1 e B1), conforme expresso na Tabela 1. Mesmo após a exclusão dos casos extremos (i.e., zero dia entre submissão e aceite do manuscrito ou mais de 1.200 dias; $n=14$ ), as diferenças entre as médias das revistas nos diferentes Qualis se mantiveram na mesma direção.

\section{Tabela 1}

Média de Tempo (em dias) entre as Datas de Submissão e de Aceite dos Manuscritos em Função do Estrato Qualis das Revistas

\begin{tabular}{cccccccc}
\hline $\begin{array}{c}\text { Qualis da revista } \\
\text { em que o artigo } \\
\text { foi publicado }\end{array}$ & $n$ & $M$ & $D P$ & Mínimo & Máximo & ANOVA & $d$ de Cohen \\
\cline { 2 - 7 } & 190 & 372,27 & 264,83 & 8 & 1240 & A $1-\mathrm{A} 2=0,49$ \\
A1 & 1052 & 257,14 & 201,97 & 0 & 1359 & $F=83,57 ;$ & A2-B $1=0,32$ \\
A2 & 1338 & 198,82 & 159,55 & 5 & 1492 & A $1-\mathrm{B} 1=0,79$ \\
B1 & 2580 & 235,37 & 192,95 & 0 & 1492 & \\
\hline Total & & & & & & \\
\hline
\end{tabular}

A Tabela 2 apresenta um ranking das revistas dentro de cada estrato Qualis avaliado pela média de tempo em dias entre as datas declaradas de submissão e aceitação dos manuscritos. Os nomes dos periódicos foram substituídos por identificadores numéricos na tabela.

O número de membros no corpo editorial da revista apresentou uma correlação negativa estatisticamente significativa com o tempo para aceitação do manuscrito $(p<0,001)$, indicando que um maior número de pessoas no corpo editorial estaria associado a um menor tempo entre a sub- missão e aceitação de um manuscrito na revista. No entanto, o tamanho de efeito desta correlação foi baixo $(r=-0,123)$.

Quando analisado o tipo de instituição a que estão vinculadas as revistas, foi encontrada uma média de tempo significativamente menor $(F=$ $14,05 ; p<0,001)$ para aceitação do manuscrito em revistas de associações/conselhos $(M=$ $207,82$ dias; $D P=171,31)$ em comparação a revistas de universidades/faculdades públicas $(M=$ $246,89 ; D P=203,69)$ ou privadas $(M=258,98$; $D P=193,58)$, mas os tamanhos de efeito $(d$ de 
Tabela 2

Ranking das Revistas por Estrato Qualis em Ordem Crescente de Média de Tempo (em dias) entre as Datas de Submissão e de Aceite dos Manuscritos

\begin{tabular}{|c|c|c|c|c|c|c|}
\hline & ID Revista & $n$ & $M$ & $D P$ & Mínimo & Máximo \\
\hline \multirow{3}{*}{ A1 } & 2 & 88 & 246,60 & 142,72 & 8 & 771 \\
\hline & 1 & 52 & 375,83 & 182,11 & 150 & 1080 \\
\hline & 3 & 50 & 589,76 & 351,00 & 110 & 1240 \\
\hline \multirow{19}{*}{ A2 } & 7 & 73 & 59,71 & 82,57 & 0 & 408 \\
\hline & 20 & 38 & 77,92 & 44,02 & 10 & 233 \\
\hline & 18 & 51 & 127,12 & 86,14 & 16 & 340 \\
\hline & 4 & 36 & 154,25 & 156,76 & 24 & 839 \\
\hline & 11 & 42 & 158,93 & 144,66 & 6 & 799 \\
\hline & 8 & 52 & 193,46 & 229,82 & 7 & 1026 \\
\hline & 16 & 34 & 211,26 & 96,00 & 2 & 492 \\
\hline & 22 & 57 & 213,21 & 152,85 & 14 & 727 \\
\hline & 19 & 71 & 220,37 & 110,09 & 13 & 550 \\
\hline & 5 & 40 & 224,80 & 115,29 & 72 & 523 \\
\hline & 13 & 66 & 239,58 & 154,01 & 40 & 807 \\
\hline & 6 & 57 & 241,30 & 104,86 & 83 & 546 \\
\hline & 9 & 51 & 243,80 & 185,70 & 13 & 747 \\
\hline & 15 & 58 & 341,78 & 226,69 & 13 & 1087 \\
\hline & 17 & 58 & 347,31 & 154,95 & 72 & 800 \\
\hline & 14 & 103 & 361,07 & 198,17 & 19 & 929 \\
\hline & 12 & 68 & 413,63 & 193,24 & 112 & 1116 \\
\hline & 10 & 41 & 427,95 & 231,79 & 15 & 1043 \\
\hline & 21 & 56 & 457,46 & 280,15 & 53 & 1359 \\
\hline \multirow{22}{*}{ B1 } & 23 & 49 & 90,27 & 62,00 & 10 & 309 \\
\hline & 40 & 99 & 96,85 & 53,25 & 24 & 446 \\
\hline & 42 & 41 & 113,41 & 53,71 & 32 & 257 \\
\hline & 43 & 92 & 142,87 & 88,09 & 15 & 515 \\
\hline & 24 & 86 & 143,28 & 64,54 & 30 & 426 \\
\hline & 28 & 59 & 148,32 & 87,74 & 15 & 359 \\
\hline & 25 & 56 & 150,55 & 138,86 & 5 & 545 \\
\hline & 32 & 54 & 155,61 & 49,37 & 108 & 472 \\
\hline & 39 & 140 & 172,55 & 91,27 & 38 & 541 \\
\hline & 36 & 40 & 176,20 & 67,11 & 23 & 396 \\
\hline & 29 & 46 & 193,59 & 132,62 & 28 & 547 \\
\hline & 38 & 22 & 209,82 & 188,47 & 52 & 854 \\
\hline & 37 & 42 & 211,62 & 77,02 & 60 & 441 \\
\hline & 41 & 40 & 227,05 & 92,11 & 24 & 445 \\
\hline & 26 & 67 & 246,18 & 213,57 & 11 & 1015 \\
\hline & 35 & 40 & 248,10 & 117,12 & 53 & 763 \\
\hline & 31 & 62 & 248,61 & 98,11 & 61 & 487 \\
\hline & 44 & 72 & 251,15 & 149,25 & 30 & 596 \\
\hline & 34 & 71 & 253,48 & 130,78 & 5 & 613 \\
\hline & 33 & 60 & 275,90 & 122,51 & 42 & 583 \\
\hline & 27 & 37 & 340,14 & 267,31 & 19 & 1004 \\
\hline & 30 & 63 & 421,52 & 379,23 & 26 & 1492 \\
\hline
\end{tabular}


Cohen) dessas diferenças foram pequenos $(d=$ 0,21 para revistas de associações versus de universidades públicas; e $d=0,28$ para revistas de associações versus de universidades privadas). Não houve diferença entre as médias de tempo para aceitação nas revistas de universidades/faculdades públicas e de universidades/faculdades privadas.

\section{Discussão}

Os objetivos deste estudo foram o de estimar o tempo médio entre a submissão/aceitação de manuscritos, que culmina na publicação de artigos nas revistas científicas mais bem avaliadas em Psicologia no Brasil, bem como investigar possíveis associações entre variáveis do processo editorial relacionado às revistas científicas. Por meio das análises realizadas, foi possível responder a esses objetivos, bem como levantar aspectos relacionados ao processo editorial, de uma amostra de revistas Qualis A1, A2 e B1 em Psicologia.

As 44 revistas analisadas possuem média de idade de 16,34 anos $(D P=11,97)$, ou seja, podem ser consideradas revistas experientes. Diferentemente de revistas mais jovens, revistas experientes tendem a ter membros editoriais com maior conhecimento do processo editorial, o que poderia facilitá-lo. A quantidade média de membros editoriais foi de 53,32 $(D P=30,37)$. Esse pode ser considerado um alto número e, assim como verificado neste estudo, espera-se que quanto maior a quantidade de membros, mais ágil seja o processo editorial, embora estes não sejam os únicos consultados, uma vez que as revistas também solicitam pareceres a consultores ad hoc. A maioria das revistas $(n=24)$ consultadas está associada a universidades e faculdades públicas e localizada na região sudeste $(n=32)$. Tendo em vista que essas instituições públicas recebem apoio financeiro público, é esperado que busquem meios de contribuir com a sociedade científica e em geral, sendo a publicação de uma revista uma possibilidade para tal (Sabadini et al., 2009). A predominância de revistas na região sudeste é coerente com a predominância de instituições de ensino superior nessa região (i.e., $50,7 \%$ dos programas de pós graduação do país; CAPES, 2013). Em relação aos idiomas de publicação, a maioria das revistas $(n=29)$ aceita artigos em três ou mais idiomas, o que evidencia a atual mobilização da comunidade científica relativa à internacionalização do conhecimento produzido no país.

Em relação à diferença entre o tempo de submissão e aceite entre os diferentes estratos, é possível que esse resultado esteja relacionado a um maior rigor adotado pelas revistas dos estratos mais altos, à visibilidade destas revistas e uma maior complexidade dos manuscritos submetidos. Ao se publicar artigos em revistas dos estratos mais altos, os pesquisadores e programas de pós-graduação recebem melhores avaliações e têm seus trabalhos mais facilmente divulgados, promovendo maior visibilidade a eles. Este fato pode fazer com que o número de submissões de manuscritos a estas revistas seja mais alto que as dos estratos mais baixos. Pode, ainda, haver uma tendência de que os manuscritos submetidos às revistas de estratos mais altos sejam mais complexos, demandando maior tempo de revisão e pareceristas mais especializados. Estes fatores aumentariam a demanda de trabalho dos editores e, consequentemente, o tempo necessário para o processo editorial. Essas hipóteses podem ser testadas caso se consiga acesso às informações referentes ao número médio de manuscritos submetidos em cada revista. Neste estudo, buscou-se acessar tais informações por meio do envio de solicitações por e-mail aos editores das revistas, porém, tal tentativa não foi exitosa. Não houve resposta às solicitações na maioria dos casos, possivelmente devido à alta demanda de trabalho dos editores. Futuros estudos nos quais se tenha acesso a essas informações podem utilizá-las para verificar a proporção entre manuscritos rejeitados e aceitos, investigando, assim, o rigor das revistas e a preferência dos autores em relação aos estratos das revistas para a submissão de seus manuscritos.

Ao se considerar todos os artigos e revistas analisados, o tempo médio declarado entre a submissão do manuscrito e o seu aceite foi de aproximadamente oito meses. Mesmo refinando a análise com a utilização da data da primeira 
revisão do manuscrito, esse intervalo é longo (i.e., aproximadamente sete meses). Esse resultado indica que mesmo considerando o papel dos autores na agilidade do processo editorial (e.g., casos em que são solicitadas revisões), o tempo médio para o aceite ainda permanece alto. Embora não se possa afirmar que a agilidade no processo editorial dependa somente da revista, este resultado sugere que os autores têm buscado atender às solicitações das revistas brevemente. O intervalo máximo de dias entre a submissão e o aceite foi de 1.492 dias, ou seja, aproximadamente quatro anos, enquanto que o mínimo foi de zero dia $(n=7)$. Este resultado relacionado ao aceite imediato de um manuscrito é questionável quando se consideram os preceitos do processo editorial, pois este requer tempo para que o manuscrito seja enviado a revisores e esses, por sua vez, demandam tempo para analisá-los.

De acordo com a APA (2010), o processo editorial tende a se prolongar por dois a três meses, sendo que esse intervalo pode ser influenciado por aspectos como o tamanho e complexidade do manuscrito, bem como pelo número de revisores envolvidos. O intervalo médio do processo editorial verificado na amostra deste estudo foi de aproximadamente oito meses, ou seja, muito maior do que aquele estimado pela APA. Estes são resultados preocupantes, tendo em vista a necessidade de se divulgar o conhecimento cientifico de forma atual. A espera por um processo editorial longo pode tornar os resultados do estudo obsoletos ou deixar de impactar a área de conhecimento ao qual se destinam. Além disto, pode impactar a avaliação do próprio periódico pela SciELO e pelo PEPSIC, uma vez que o tempo de processamento dos artigos é um critério de qualidade avaliado por estes indexadores. A indicação da profissionalização da gestão editorial como um dos desafios da SciELO pode estar relacionada à morosidade na avaliação de manuscritos verificada no presente estudo.

A demora na avaliação dos manuscritos tem efeitos claros para indicadores de fator de impacto das revistas e fator $h$ das revistas e dos pesquisadores. Tais índices levam em consideração o número de citações feitas em um dado período de tempo a um artigo ou autor(a). Quando o ma- terial publicado está desatualizado por conta do tempo entre submissão e aceitação (e posterior publicação), a probabilidade de ser citado diminui. A demora na citação dos artigos acaba por superar o tempo necessário para causar impacto neste fator de avaliação, além da publicação de artigos com referências desatualizadas.

Medidas atuais (e.g., Altmetrics) demonstram que o atraso na publicação de resultados de pesquisa prejudica a divulgação da ciência e o avanço do conhecimento na área da Psicologia. O tempo encontrado neste estudo como necessário para a aceitação de um manuscrito, acrescido a um tempo necessário para posterior publicação é, muitas vezes, maior do que o tempo para publicação de um livro em Psicologia no Brasil. Por isto são necessárias intervenções que subsidiem mudanças drásticas nesse panorama atual das melhores revistas de Psicologia do país.

Outras variáveis não investigadas no presente estudo, tais como o tamanho e a complexidade do manuscrito e/ou número de revisores envolvidos (APA, 2010), podem influenciar o processo editorial. Tendo em vista que o tamanho do manuscrito geralmente é padronizado pela revista, considera-se que esta não seja uma variável que possa impactar o processo editorial. Em relação à complexidade do manuscrito, tal variável demanda um julgamento de valor que não fez parte do escopo deste estudo. $\mathrm{O}$ número de revisores envolvidos no processo editorial de cada manuscrito é uma informação que não é divulgada pelas revistas embora usualmente adotem o sistema de revisão por dois revisores, o qual é um requisito da CAPES e da SciELO para a avaliação dos periódicos. As revistas poderiam, além das datas de submissão, revisões e aceite, divulgar o número de revisores envolvidos nos processos editoriais de cada artigo disponibilizando, assim, mais informações para a realização de futuras análises.

Dentre as variáveis analisadas, constatou-se que quanto maior o número de membros do corpo editorial, menor tende a ser o tempo decorrido entre submissão e aceitação de um manuscrito, embora o tamanho do efeito dessa relação tenha sido baixo $(r=-0,123)$. Tal resultado é logicamente compreensível uma vez que um 
maior número de membros do corpo editorial resulta em maior número de pessoas (e.g., revisores, editores, secretários) comprometidas com o andamento do processo editorial. Por outro lado, o baixo número de membros do corpo editorial pode dificultar o processo editorial, pois estes acabam acumulando funções e/ou não há alternativas suficientes de revisores para a demanda de manuscritos recebida.

Embora o maior número de membros do corpo editorial possa ser uma variável a se considerar com o objetivo de tornar o processo editorial mais ágil, há de se considerar também o desafio de mobilizar a comunidade científica para fazer parte do corpo editorial ou da comissão de consultores ad hoc de uma revista científica. Estas são tarefas que demandam tempo e trabalho e não resultam em retorno financeiro direto. No Brasil, a carreira acadêmica acumula diversas funções de pesquisa, extensão e docência, além de atividades burocráticas relacionadas, por exemplo, a relatórios e prestações de contas para agências de fomento. A rotina dos acadêmicos inclui reuniões de departamento e grupos de pesquisa, supervisões de estudantes e atividades de ensino, escrita de propostas para editais de pesquisa, realização de pesquisas, escrita de artigos e capítulos de livro, revisões de artigos, preparação de palestras e viagens a congressos, entre diversas outras atividades. Todas essas funções tornam bastante custoso manejar o tempo dentro da academia (Couto, 2014). Diante deste panorama, alguns profissionais tendem a evitar a acumulação de funções "extras", sendo aquelas relacionadas às revistas encaradas como tal. Uma alternativa aplicada por alguns periódicos é o convite a estudantes de doutorado para realizar a revisão de artigos científicos. Outra possível alternativa incluiria a adoção de um processo de cadastramento espontâneo de revisores para este trabalho nos sites das revistas, aliado à implantação de um treinamento de futuros revisores, de forma a padronizar e agilizar o processo de avaliação dos manuscritos. Este treinamento poderia ser automatizado, no estilo de vídeo aulas, por exemplo, ou os programas de pós-graduação poderiam inseri-lo em sua grade curricular.
Foi verificado, ainda, que as revistas vinculadas às associações e conselhos tendem a ser mais ágeis do que as demais (i.e., vinculadas a universidade/ faculdades públicas ou privadas). Embora os resultados do presente estudo não forneçam informação suficiente para compreender o motivo dessa diferença, algumas hipóteses podem ser levantadas: (a) tais revistas podem contar com a colaboração de profissionais exercendo cargos outros que não o de professores em universidades, acumulando assim menos funções em seus trabalhos e podendo dar retorno mais rápido às submissões em suas revistas; (b) devido ao fato de não serem filiadas a programas de pós-graduação, estas revistas podem não ser muito divulgadas nesse meio e, consequentemente, não procuradas frequentemente para a submissão de manuscritos por pesquisadores, resultando em menor número de publicações e maior agilidade no processo editorial.

Embora este estudo tenha fornecido informações sobre possíveis variáveis relacionadas à agilidade do processo editorial de revistas Qualis A1, A2 e B1 em Psicologia, os resultados apresentados devem ser utilizados com cautela diante de suas limitações. As análises realizadas foram baseadas nas datas declaradas pelas próprias revistas nos artigos. Embora essas informações devam ser fidedignas, muitas vezes elas podem não refletir a realidade. É de conhecimento da comunidade científica, mesmo que informalmente, situações nas quais autores não concordam com as datas publicadas nos artigos, pois estas não refletem o (longo) período de tempo do processo editorial. Além disto, podem ocorrer situações nas quais a revista solicita aos autores que ressubmetam o manuscrito devido ao longo período que este permaneceu no processo editorial. Diante da nova submissão, o processo editorial tende a ser ágil, porém, as datas declaradas não condizem com todo o tempo demandado para a publicação.

Outras limitações deste estudo se referem à restrição estabelecida para a coleta de dados. Devido ao amplo número de periódicos científicos e, consequentemente, de artigos publicados, foi necessário estabelecer um recorte. Foram analisados artigos de periódicos científicos perten- 
centes aos estratos A1 e A2 em sua totalidade e parte dos periódicos científicos do estrato B1, não abrangendo, assim, a totalidade de periódicos e artigos científicos da área de Psicologia existentes atualmente. Além disto, não se buscou avaliar o tempo entre a aceitação do manuscrito e a disponibilização (publicação propriamente dita) do artigo em um dado número da revista. Este processo muitas vezes leva tempo, podendo tornar ainda maior o espaço entre a submissão de um trabalho científico e a divulgação dos seus resultados para a comunidade científica em formato de artigo, estendendo-se em longas "filas de espera".

É necessário que as revistas se adequem às necessidades do meio científico para melhor atender as demandas de publicações. $\mathrm{O}$ esquema de publicações sequenciais (e.g., semestrais, quadrimestrais) pode, por exemplo, ser complementado por formas de veiculação dos artigos mais diretas após a aceitação, como é o caso de revistas que utilizam pré-prints ou processos de online first. Esta alternativa, no entanto, somente auxilia na diminuição do tempo entre a aceitação e a publicação do artigo (não avaliado neste estudo). Faltam ainda processos similares que auxiliem a diminuir este tempo.

Um dos objetivos da mensuração de índices de avaliação para a pesquisa científica é oferecer informação para direcionar o uso de recursos para pesquisa - públicos e privados. Os trabalhos em cientometria buscam desenvolver critérios cada vez mais elaborados para tornar essa mensuração o mais eficaz possível (Pinto, 2008). No entanto, o prejuízo que o atraso na publicação de artigos pode gerar às avaliações de revista, de instituições e de pesquisadores, concernente ao fator de impacto e fator $h$, sugerem fortemente a necessidade de indicadores bibliométricos qualitativos em cientometria (Silva \& Bianchi, 2001). A utilização única de indicadores quantitativos, tais como o número de publicações e citações, tem levado pesquisadores a submeter maior número de artigos, congestionando os processos editorais (Silva \& Bianchi, 2001). O resultado tende a ser um ciclo vicioso: pesquisadores submetem cada vez mais artigos visando o aumento de seus indicadores bibliométricos; as revistas não conseguem dar vazão ao volume de submissões em tempo hábil; artigos acabam sendo publicados com atraso, diminuindo chances de citações; pesquisadores buscam maior número de citações submetendo novos artigos, iniciando novamente o ciclo.

Embora uma crítica frequente ao uso de indicadores qualitativos na cientometria seja a inclusão de uma parcela significativa de subjetividade na avaliação do fenômeno, as limitações dos critérios quantitativos parecem exigir a complementação em parceria com estes outros critérios. Mesmo critérios já consagrados internacionalmente, como o uso do fator de impacto para avaliação de um periódico, possuem limitações subjetivas de aplicação, como a impossibilidade de comparação do alcance científico de periódicos de diferentes áreas a partir dos seus fatores de impacto, uma vez que características próprias de cada área exigem uma interpretação distinta do que seria um fator de impacto baixo, médio ou alto (Pinto, 2008). Da mesma forma, diferentes tipos de manuscritos (e.g., estudo empírico, estudo teórico, revisão de literatura) possuem diferentes potenciais de citação, por exemplo (Silva \& Bianchi, 2001). Assim, características qualitativas da área da ciência, do tipo de estudo, dos pesquisadores e do contexto em que se encontram (e.g. instituição privada ou pública, país de procedência) inegavelmente influenciam os indicadores da produção científica, o que torna no mínimo ingênuo negligenciar a necessidade de se investir em avaliações qualitativas complementares de produtividade científica.

Mesmo diante das limitações do presente estudo, foi possível verificar algumas variáveis relacionadas ao tempo despendido no processo editorial de revistas Qualis A1, A2 e B1 em Psicologia. Os resultados apresentados podem servir de embasamento para a busca de maior agilidade dentre as revistas. Estudos futuros podem ser conduzidos buscando ampliar a amostra de artigos analisados, bem como incluir demais variáveis (e.g., número de revisores, média de submissões por revista) nas análises realizadas e aprofundar o entendimento sobre alguns resultados deste estudo. 


\section{Referências}

American Psychological Association. (2010). Publication manual of the American Psychological Association (6 ${ }^{\text {th }}$ ed.). Washington, DC: Author.

Coordenação de Aperfeiçoamento de Pessoal de Nível Superior. (2013). Documento Critério Qualis por Área 2013 (Psicologia). Recuperado em http://qualis.capes.gov.br/webqualis/publico/ documentosDeArea.seam?conversationPropaga tion=begin

Coordenação de Aperfeiçoamento de Pessoal de Nível Superior. (n.d.). Documento Consulta por Classificação / Área Avaliação (Psicologia). Recuperado em http://qualis.capes.gov.br/webqualis/publico/pesquisaPublicaClassificacao. seam

Couto, M. C. P. P. (2014). Como manejar o tempo na academia. In S. H. Koller, M. C. P. P. Couto, \& J. V. Hohendorff (Eds.), Manual de produção científica (pp. 167-177). Porto Alegre, RS: Penso.

Hutz, C. S., Rocha, M. L., Spink, M. J. P., \& Menandro, P. R. M. (2010). Perfil, avaliação e metas de produção intelectual dos programas de pós-graduação em psicologia. Psicologia: Reflexão e Crítica, 23(1), 25-34.

Montanari, F., \& Packer, A. L. (2014). Critérios de seleção de periódicos para indexação e publicação nas coleções da rede SciELO. In A. L. Packer, N. Cop, A. Luccisano, A. Ramalho, \& E. Spinak (Eds.), Scielo - 15 anos de acesso aberto: Um estudo analítico sobre acesso aberto $e$ comunicação cientifica (pp. 67-80). Paris: Organização das Nações Unidas para a Educação, a Ciência e a Cultura.

Packer, A. L., \& Meneghini, R. (2014). O Scielo aos 15 anos: Raison d'être, avanços e desafios para o futuro. In A. L. Packer, N. Cop, A. Luccisano, A. Ramalho, \& E. Spinak (Eds.), Scielo - 15 anos de acesso aberto: Um estudo analítico sobre acesso aberto e comunicação cientifica (pp. 15-28). Paris: Organização das Nações Unidas para a Educação, a Ciência e a Cultura.

Pinto, L. A. (2008). Cientometria: É possível avaliar qualidade da pesquisa científica? [Editorial]. Scientia Medica, 18(2), 64-65.

Sabadini, A. A. Z. P., Sampaio, M. I. C., \& Nascimento, M. M. (2009). Preparando um periódico científico. In A. A. Z. P. Sabadini, M. I. C. Sam- paio, \& S. H. Koller (Eds.), Publicar em Psicologia: Um enfoque para a revista científica (pp. 35-73). São Paulo, SP: Associação Brasileira de Editores Científicos de Psicologia.

Silva, J. A., \& Bianchi, M. L. P. (2001). Cientometria: A métrica da ciência. Paidéia (Ribeirão Preto), 11(20), 5-10. doi:10.1590/S0103$-863 X 2001000200002$

Thomaz, P. G., Assad, R. S., \& Moreira, L. F. P. (2011). Uso do fator de impacto e do índice $\mathrm{H}$ para avaliar pesquisadores e publicações. Arquivos Brasileiros de Cardiologia, 96(2), 90-93. doi:10.1590/S0066-782X2011000200001

Trzesniak, P., \& Koller, S. H. (2009). A redação científica apresentada por editores. In A. A. Z. P. Sabadini, M. I. C. Sampaio, \& S. H. Koller (Eds.), Publicar em Psicologia: Um enfoque para a revista cientifica (pp. 19-33). São Paulo, SP: Associação Brasileira de Editores Científicos de Psicologia.

Wendt, G. G., Lisboa, C. S. M., DeSousa, D. A., \& Koller, S. H. (2013). Perfil dos bolsistas de produtividade em pesquisa do CNPq em Psicologia. Psicologia: Ciência e Profissão, 33(3), 536-547. doi:10.1590/S1414-98932013000300003

Yamamoto, O. H., \& Costa, A. L. F. (2009). A avaliação de periódicos científicos brasileiros da área da Psicologia. In A. A. Z. P. Sabadini, M. I. C. Sampaio, \& S. H. Koller (Eds.), Publicar em Psicologia: Um enfoque para a revista cientifica (pp. 189-199). São Paulo, SP: Associação Brasileira de Editores Científicos de Psicologia.

Yamamoto, O. H., Tourinho, E. Z., Bastos, A. V. B., \& Menandro, P. R. M. (2012). Produção científica e "produtivismo": Há alguma luz no final do túnel? Revista Brasileira de Pós-Graduação, 9(18), 727-750. 